\title{
Quantum Field Theory of boson mixing
}

\author{
Massimo Blasone ${ }^{\sharp b}$, Antonio Capolupo ${ }^{b}$, Oreste Romei ${ }^{b}$ and Giuseppe Vitiello ${ }^{b}$ \\ ${ }^{\dagger}$ Blackett Laboratory, Imperial College, Prince Consort Road, London SW7 2BZ, U.K. \\ b Dipartimento di Fisica and INFN, Università di Salerno, I-84100 Salerno, Italy
}

\begin{abstract}
We consider the quantum field theoretical formulation of boson field mixing and obtain the exact oscillation formula. This formula does not depend on arbitrary mass parameters. We show that the space for the mixed field states is unitarily inequivalent to the state space where the unmixed field operators are defined. We also study the structure of the currents and charges for the mixed fields.
\end{abstract}

\section{INTRODUCTION}

Particle mixing and oscillations (for a recent review, see Ref. [1]) are among the most intriguing topics of Particle Physics. The mixing of neutrinos and their oscillations seem to be now experimentally established after a long search [2]. On the other hand, quark mixing and meson mixing are widely accepted and verified [3]. However, many features of the physics of mixing are still obscure, for example the issue related to its origin in the context of Standard Model and the related problem of the generation of masses [4].

Also from a purely mathematical point of view, there are aspects which are not fully understood. Indeed, only recently [5] a rich non-perturbative vacuum structure has been discovered to be associated with the mixing of fermion fields in the context of Quantum Field Theory (for a mathematically rigorous approach see Ref. [6]). The careful study of such a structure [7] has led to the determination of the exact QFT formula for neutrino oscillations [8,9], exhibiting new features with respect to the usual quantum mechanical Pontecorvo formula [10. Actually, it turns out 11.12] that the non-trivial nature of the mixing transformations manifests itself also in the case of the mixing of boson fields. Of course, in this case the condensate structure for the "flavor" vacuum is very much different from the fermion case and a careful analysis is necessary in order to understand which phenomenological consequences are to be expected for the oscillations of mixed bosons.

In this paper, we perform this analysis first at a formal level and then we study the oscillations of mixed mesons of the kind of the $K^{0}-\bar{K}^{0}$. We will treat these particles as stable ones, an approximation which however does not affect the general validity of our results. In the framework of the QFT analysis of Refs. [5, 11], a study of the meson mixing and oscillations has been carried out in Ref. [12], where modifications to the usual oscillation formulas, connected with the vacuum structure, have been presented. However, the results of Ref. [12] can be improved in many respects and in the present paper we show that the oscillation formula there obtained has to be actually replaced with the exact one here presented.

In Section II we study the quantum field theory of two mixed spin-zero boson fields. In Section III we analyze the structure of currents for mixed fields and we derive the exact oscillation formula in Section IV. Section V is devoted to conclusions. Some mathematical derivations are given in the Appendix.

\section{MIXING OF BOSON FIELDS IN QFT}

The observed boson oscillations always involve particles with zero electrical charge. What oscillate are some other quantum numbers such as the strangeness and the isospin. Therefore, in the study of boson mixing we have to consider always 13] complex fields. The charge in question is some "flavor charge" (e.g. the strangeness) and thus the complex fields are "flavor charged" fields, referred to as "flavor fields" for simplicity.

We define the mixing relations as:

$$
\begin{aligned}
& \phi_{A}(x)=\phi_{1}(x) \cos \theta+\phi_{2}(x) \sin \theta \\
& \phi_{B}(x)=-\phi_{1}(x) \sin \theta+\phi_{2}(x) \cos \theta
\end{aligned}
$$

where generically we denote the mixed fields with suffixes $A$ and $B$. Let the fields $\phi_{i}(x), i=1,2$, be free complex fields with definite masses. Their conjugate momenta are $\pi_{i}(x)=\partial_{0} \phi_{i}^{\dagger}(x)$ and the commutation relations are the usual ones: 


$$
\left[\phi_{i}(x), \pi_{j}(y)\right]_{t=t^{\prime}}=\left[\phi_{i}^{\dagger}(x), \pi_{j}^{\dagger}(y)\right]_{t=t^{\prime}}=i \delta^{3}(\mathbf{x}-\mathbf{y}) \delta_{i j} \quad, \quad i, j=1,2 .
$$

with the other equal-time commutators vanishing. The Fourier expansions of fields and momenta are:

$$
\begin{gathered}
\phi_{i}(x)=\int \frac{d^{3} k}{(2 \pi)^{\frac{3}{2}}} \frac{1}{\sqrt{2 \omega_{k, i}}}\left(a_{\mathbf{k}, i} e^{-i \omega_{k, i} t}+b_{\mathbf{k}, i}^{\dagger} e^{i \omega_{k, i} t}\right) e^{i \mathbf{k} \cdot \mathbf{x}} \\
\pi_{i}(x)=i \int \frac{d^{3} k}{(2 \pi)^{\frac{3}{2}}} \sqrt{\frac{\omega_{k, i}}{2}}\left(a_{\mathbf{k}, i}^{\dagger} e^{i \omega_{k, i} t}-b_{\mathbf{k}, i} e^{-i \omega_{k, i} t}\right) e^{i \mathbf{k} \cdot \mathbf{x}},
\end{gathered}
$$

where $\omega_{k, i}=\sqrt{\mathbf{k}^{2}+m_{i}^{2}}$ and $\left[a_{\mathbf{k}, i}, a_{\mathbf{p}, j}^{\dagger}\right]=\left[b_{\mathbf{k}, i}, b_{\mathbf{p}, j}^{\dagger}\right]=\delta^{3}(\mathbf{k}-\mathbf{p}) \delta_{i j}$, with $i, j=1,2$ and the other commutators vanishing. We will consider stable particles, which will not affect the general validity of our results.

We now proceed in a similar way to what has been done in Ref. [5] for fermions and recast Eqs. (1) into the form:

$$
\begin{aligned}
& \phi_{A}(x)=G_{\theta}^{-1}(t) \phi_{1}(x) G_{\theta}(t) \\
& \phi_{B}(x)=G_{\theta}^{-1}(t) \phi_{2}(x) G_{\theta}(t)
\end{aligned}
$$

and similar ones for $\pi_{A}(x), \pi_{B}(x)$. $G_{\theta}(t)$ denotes the operator which implements the mixing transformations (1):

$$
G_{\theta}(t)=\exp \left[-i \theta \int d^{3} x\left(\pi_{1}(x) \phi_{2}(x)-\phi_{1}^{\dagger}(x) \pi_{2}^{\dagger}(x)-\pi_{2}(x) \phi_{1}(x)+\phi_{2}^{\dagger}(x) \pi_{1}^{\dagger}(x)\right)\right],
$$

which is (at finite volume) a unitary operator: $G_{\theta}^{-1}(t)=G_{-\theta}(t)=G_{\theta}^{\dagger}(t)$. The generator of the mixing transformation in the exponent of $G_{\theta}(t)$ can also be written as

$$
G_{\theta}(t)=\exp \left[\theta\left(S_{+}(t)-S_{-}(t)\right)\right] .
$$

The operators

$$
S_{+}(t)=S_{-}^{\dagger}(t) \equiv-i \int d^{3} x\left(\pi_{1}(x) \phi_{2}(x)-\phi_{1}^{\dagger}(x) \pi_{2}^{\dagger}(x)\right)
$$

together with

$$
\begin{gathered}
S_{3} \equiv \frac{-i}{2} \int d^{3} x\left(\pi_{1}(x) \phi_{1}(x)-\phi_{1}^{\dagger}(x) \pi_{1}^{\dagger}(x)-\pi_{2}(x) \phi_{2}(x)+\phi_{2}^{\dagger}(x) \pi_{2}^{\dagger}(x)\right) \\
S_{0}=\frac{Q}{2} \equiv \frac{-i}{2} \int d^{3} x\left(\pi_{1}(x) \phi_{1}(x)-\phi_{1}^{\dagger}(x) \pi_{1}^{\dagger}(x)+\pi_{2}(x) \phi_{2}(x)-\phi_{2}^{\dagger}(x) \pi_{2}^{\dagger}(x)\right),
\end{gathered}
$$

close the $s u(2)$ algebra (at each time $t):\left[S_{+}(t), S_{-}(t)\right]=2 S_{3},\left[S_{3}, S_{ \pm}(t)\right]= \pm S_{ \pm}(t),\left[S_{0}, S_{3}\right]=\left[S_{0}, S_{ \pm}(t)\right]=0$. Note that $S_{3}$ and $S_{0}$ are time independent. It is useful to write down explicitly the expansions of the above generators in terms of annihilation and creation operators:

$$
\begin{gathered}
S_{+}(t)=\int d^{3} k\left(U_{\mathbf{k}}^{*}(t) a_{\mathbf{k}, 1}^{\dagger} a_{\mathbf{k}, 2}-V_{\mathbf{k}}^{*}(t) b_{-\mathbf{k}, 1} a_{\mathbf{k}, 2}+V_{\mathbf{k}}(t) a_{\mathbf{k}, 1}^{\dagger} b_{-\mathbf{k}, 2}^{\dagger}-U_{\mathbf{k}}(t) b_{-\mathbf{k}, 1} b_{-\mathbf{k}, 2}^{\dagger}\right) \\
S_{-}(t)=\int d^{3} k\left(U_{\mathbf{k}}(t) a_{\mathbf{k}, 2}^{\dagger} a_{\mathbf{k}, 1}-V_{\mathbf{k}}(t) a_{\mathbf{k}, 2}^{\dagger} b_{-\mathbf{k}, 1}^{\dagger}+V_{\mathbf{k}}^{*}(t) b_{-\mathbf{k}, 2} a_{\mathbf{k}, 1}-U_{\mathbf{k}}^{*}(t) b_{-\mathbf{k}, 2} b_{-\mathbf{k}, 1}^{\dagger}\right) \\
S_{3}=\frac{1}{2} \int d^{3} k\left(a_{\mathbf{k}, 1}^{\dagger} a_{\mathbf{k}, 1}-b_{-\mathbf{k}, 1}^{\dagger} b_{-\mathbf{k}, 1}-a_{\mathbf{k}, 2}^{\dagger} a_{\mathbf{k}, 2}+b_{-\mathbf{k}, 2}^{\dagger} b_{-\mathbf{k}, 2}\right) \\
S_{0}=\frac{1}{2} \int d^{3} k\left(a_{\mathbf{k}, 1}^{\dagger} a_{\mathbf{k}, 1}-b_{-\mathbf{k}, 1}^{\dagger} b_{-\mathbf{k}, 1}+a_{\mathbf{k}, 2}^{\dagger} a_{\mathbf{k}, 2}-b_{-\mathbf{k}, 2}^{\dagger} b_{-\mathbf{k}, 2}\right) .
\end{gathered}
$$


As for the case of the fermion mixing, the structure of the generator Eq.(7) is recognized to be the one of a rotation combined with a Bogoliubov transformation (see below Eqs.(21)-(24)). Indeed, in the above equations, the coefficients $U_{\mathbf{k}}(t) \equiv\left|U_{\mathbf{k}}\right| e^{i\left(\omega_{k, 2}-\omega_{k, 1}\right) t}$ and $V_{\mathbf{k}}(t) \equiv\left|V_{\mathbf{k}}\right| e^{i\left(\omega_{k, 1}+\omega_{k, 2}\right) t}$ appear to be the Bogoliubov coefficients. They are defined as

$$
\left|U_{\mathbf{k}}\right| \equiv \frac{1}{2}\left(\sqrt{\frac{\omega_{k, 1}}{\omega_{k, 2}}}+\sqrt{\frac{\omega_{k, 2}}{\omega_{k, 1}}}\right), \quad\left|V_{\mathbf{k}}\right| \equiv \frac{1}{2}\left(\sqrt{\frac{\omega_{k, 1}}{\omega_{k, 2}}}-\sqrt{\frac{\omega_{k, 2}}{\omega_{k, 1}}}\right)
$$

and satisfy the relation

$$
\left|U_{\mathbf{k}}\right|^{2}-\left|V_{\mathbf{k}}\right|^{2}=1
$$

which is in fact to be expected in the boson case (note the difference with respect to the fermion case of Ref. [D] ). We can thus put $\left|U_{\mathbf{k}}\right| \equiv \cosh \xi_{1,2}^{\mathbf{k}},\left|V_{\mathbf{k}}\right| \equiv \sinh \xi_{1,2}^{\mathbf{k}}$, with $\xi_{1,2}^{\mathbf{k}}=\frac{1}{2} \ln \frac{\omega_{k, 1}}{\omega_{k, 2}}$.

We now consider the action of the generator of the mixing transformations on the vacuum $|0\rangle_{1,2}$ for the fields $\phi_{1,2}(x): a_{\mathbf{k}, i}|0\rangle_{1,2}=0, i=1,2$. The generator induces an $S U(2)$ coherent state structure on such state [14]:

$$
|0(\theta, t)\rangle_{A, B} \equiv G_{\theta}^{-1}(t)|0\rangle_{1,2} .
$$

From now on we will refer to the state $|0(\theta, t)\rangle_{A, B}$ as to the "flavor" vacuum for bosons. The suffixes $A$ and $B$ label the flavor charge content of the state. We have ${ }_{A, B}\langle 0(\theta, t) \mid 0(\theta, t)\rangle_{A, B}=1$. In the following, we will consider the Hilbert space for flavor fields at a given time $t$, say $t=0$, and it is useful to define $|0(t)\rangle_{A, B} \equiv|0(\theta, t)\rangle_{A, B}$ and $|0\rangle_{A, B} \equiv|0(\theta, t=0)\rangle_{A, B}$ for future reference. A crucial point is that the flavor and the mass vacua are orthogonal in the infinite volume limit. We indeed have (see Appendix):

$$
{ }_{1,2}\langle 0 \mid 0(t)\rangle_{A, B}=\prod_{\mathbf{k}}{ }_{1,2}\left\langle 0\left|G_{\mathbf{k}, \theta}^{-1}(t)\right| 0\right\rangle_{1,2}=\prod_{\mathbf{k}} f_{0}^{\mathbf{k}}(\theta), \quad \text { for any } t,
$$

where we have used $G_{\theta}^{-1}(t)=\prod_{\mathbf{k}} G_{\mathbf{k}, \theta}^{-1}(t)$ (see Eqs.(8), (12) and (13) ). In the infinite volume limit, we obtain

$$
\lim _{V \rightarrow \infty}{ }_{1,2}\langle 0 \mid 0(t)\rangle_{A, B}=\lim _{V \rightarrow \infty} e^{\frac{V}{(2 \pi)^{3}} \int d^{3} k \ln f_{0}^{\mathbf{k}}(\theta)}=0, \text { for any } t .
$$

From the Appendix, Eq.(56), we see that $\ln f_{0}^{\mathbf{k}}(\theta)$ is indeed negative for any values of $\mathbf{k}, \theta$ and $m_{1}, m_{2}$ (note that $0 \leq \theta \leq \pi / 4)$. We also observe that the orthogonality disappears when $\theta=0$ and/or $m_{1}=m_{2}$, consistently with the fact that in both cases there is no mixing. These features are similar to the case of fermion mixing [5]: the orthogonality is essentially due to the infinite number of degrees of freedom [15, 16] (The statement of Ref. [12 that in the boson case the above vacua are orthogonal also at finite volume has to be therefore corrected according to the present result).

We can define annihilation operators for the vacuum $|0(t)\rangle_{A, B}$ as $a_{\mathbf{k}, A}(\theta, t) \equiv G_{\theta}^{-1}(t) a_{\mathbf{k}, 1} G_{\theta}(t)$, etc.. with $a_{\mathbf{k}, A}(\theta, t)|0(t)\rangle_{A, B}=0$. For simplicity we will use the notation $a_{\mathbf{k}, A}(t) \equiv a_{\mathbf{k}, A}(\theta, t)$. Explicitly, we have:

$$
\begin{aligned}
a_{\mathbf{k}, A}(t) & =\cos \theta a_{\mathbf{k}, 1}+\sin \theta\left(U_{\mathbf{k}}^{*}(t) a_{\mathbf{k}, 2}+V_{\mathbf{k}}(t) b_{-\mathbf{k}, 2}^{\dagger}\right), \\
a_{\mathbf{k}, B}(t) & =\cos \theta a_{\mathbf{k}, 2}-\sin \theta\left(U_{\mathbf{k}}(t) a_{\mathbf{k}, 1}-V_{\mathbf{k}}(t) b_{-\mathbf{k}, 1}^{\dagger}\right), \\
b_{-\mathbf{k}, A}(t) & =\cos \theta b_{-\mathbf{k}, 1}+\sin \theta\left(U_{\mathbf{k}}^{*}(t) b_{-\mathbf{k}, 2}+V_{\mathbf{k}}(t) a_{\mathbf{k}, 2}^{\dagger}\right), \\
b_{-\mathbf{k}, B}(t) & =\cos \theta b_{-\mathbf{k}, 2}-\sin \theta\left(U_{\mathbf{k}}(t) b_{-\mathbf{k}, 1}-V_{\mathbf{k}}(t) a_{\mathbf{k}, 1}^{\dagger}\right) .
\end{aligned}
$$

These operators satisfy the canonical commutation relations (at equal times). In their expressions the Bogoliubov transformation part is evidently characterized by the terms with the $U$ and $V$ coefficients. The condensation density of the flavor vacuum is given for any $t$ by

$$
{ }_{A, B}\left\langle 0(t)\left|a_{\mathbf{k}, i}^{\dagger} a_{\mathbf{k}, i}\right| 0(t)\right\rangle_{A, B}={ }_{A, B}\left\langle 0(t)\left|b_{-\mathbf{k}, i}^{\dagger} b_{-\mathbf{k}, i}\right| 0(t)\right\rangle_{A, B}=\sin ^{2} \theta\left|V_{\mathbf{k}}\right|^{2} \quad, \quad i=1,2 .
$$

It is useful to note that $\left|V_{\mathbf{k}}\right|^{2}$ can be written as a function of the rescaled momentum $p \equiv \sqrt{\frac{2|\mathbf{k}|^{2}}{m_{1}^{2}+m_{2}^{2}}}$ and of the adimensional parameter $a \equiv \frac{m_{2}^{2}-m_{1}^{2}}{m_{1}^{2}+m_{2}^{2}}$ as follows: 


$$
|V(p, a)|^{2}=\frac{p^{2}+1}{2 \sqrt{\left(p^{2}+1\right)^{2}-a^{2}}}-\frac{1}{2},
$$

from which we see that the condensation density is maximal at $p=0\left(\left|V_{\max }\right|^{2}=\frac{\left(m_{1}-m_{2}\right)^{2}}{4 m_{1} m_{2}}\right)$ and goes to zero for large momenta (i.e. for $|\mathbf{k}|^{2} \gg \frac{m_{1}^{2}+m_{2}^{2}}{2}$ ). Note that the corresponding quantity in the fermion case is limited to the value $1 / 2$ and the momentum scale is given by $\sqrt{m_{1} m_{2}}$. A plot of $|V(p, a)|^{2}$ is presented in Fig.1 for sample values of the parameter $a$.

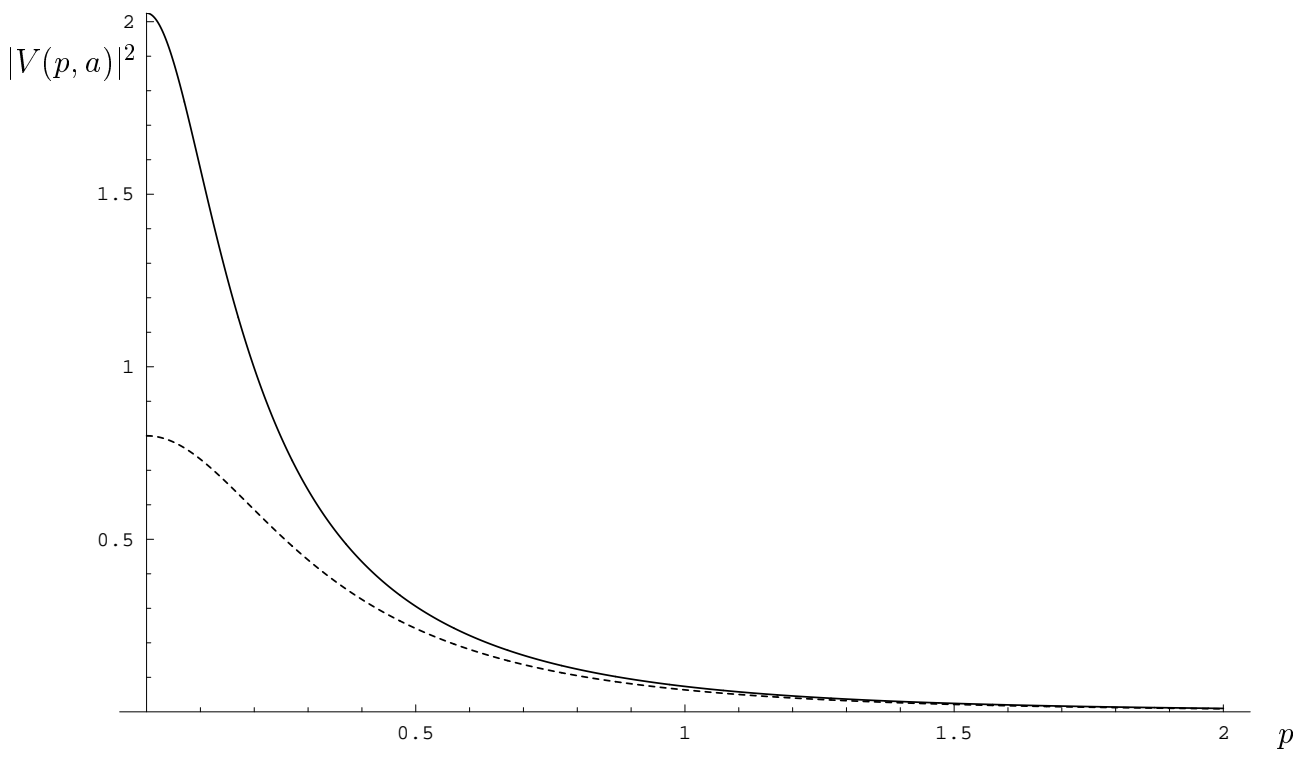

Figure 1: The condensation density $|V(p, a)|^{2}$ as a function of $p$ for $a=0.98$ (solid line) and $a=0.92$ (dashed line).

\section{A. Arbitrary mass parameterization}

Above we have expanded the mixed fields $\phi_{A, B}$ in the same basis as the free fields $\phi_{1,2}$. However, as noticed in Ref. [7] for the case of fermion mixing, this is not the most general possibility. Indeed, one could as well expand the flavor fields in a basis of fields with arbitrary masses. Of course, these arbitrary mass parameters should not appear in the physically observable quantities. Thus, as a check for the validity of the oscillation formula we are going to derive in Section IV, it is important to consider this generalization. Let us first rewrite the free fields $\phi_{1,2}$ in the form

$$
\begin{aligned}
& \phi_{i}(x)=\int \frac{d^{3} k}{(2 \pi)^{\frac{3}{2}}}\left(u_{\mathbf{k}, i}^{\phi}(t) a_{\mathbf{k}, i}+v_{-\mathbf{k}, i}^{\phi}(t) b_{-\mathbf{k}, i}^{\dagger}\right) e^{i \mathbf{k} \cdot \mathbf{x}}, \\
& \pi_{i}(x)=i \int \frac{d^{3} k}{(2 \pi)^{\frac{3}{2}}}\left(u_{\mathbf{k}, i}^{\pi}(t) a_{\mathbf{k}, i}^{\dagger}-v_{-\mathbf{k}, i}^{\pi}(t) b_{-\mathbf{k}, i}\right) e^{i \mathbf{k} \cdot \mathbf{x}} \quad, \quad i=1,2,
\end{aligned}
$$

where we have introduced the notation

$$
\begin{aligned}
& u_{\mathbf{k}, i}^{\phi}(t) \equiv \frac{1}{\sqrt{2 \omega_{k, i}}} e^{-i \omega_{k, i} t} \quad, \quad v_{-\mathbf{k}, i}^{\phi}(t) \equiv \frac{1}{\sqrt{2 \omega_{k, i}}} e^{i \omega_{k, i} t}, \\
& u_{\mathbf{k}, i}^{\pi}(t) \equiv \sqrt{\frac{\omega_{k, i}}{2}} e^{i \omega_{k, i} t} \quad, \quad v_{-\mathbf{k}, i}^{\pi}(t) \equiv \sqrt{\frac{\omega_{k, i}}{2}} e^{-i \omega_{k, i} t} \quad, \quad i=1,2 .
\end{aligned}
$$

We now define 


$$
\begin{aligned}
\rho_{\alpha \beta}^{\mathbf{k} *}(t) & \equiv u_{\mathbf{k}, \alpha}^{\pi}(t) u_{\mathbf{k}, \beta}^{\phi}(t)+v_{-\mathbf{k}, \alpha}^{\phi}(t) v_{-\mathbf{k}, \beta}^{\pi}(t)=e^{i\left(\omega_{k, \alpha}-\omega_{k, \beta}\right) t} \cosh \xi_{\alpha, \beta}^{\mathbf{k}}, \\
\lambda_{\alpha \beta}^{\mathbf{k} *}(t) & \equiv v_{-\mathbf{k}, \alpha}^{\pi}(t) u_{\mathbf{k}, \beta}^{\phi}(t)-u_{\mathbf{k}, \alpha}^{\phi}(t) v_{-\mathbf{k}, \beta}^{\pi}(t)=e^{-i\left(\omega_{k, \alpha}+\omega_{k, \beta}\right) t} \sinh \xi_{\alpha, \beta}^{\mathbf{k}}, \\
\xi_{\alpha, \beta}^{\mathbf{k}} & \equiv \frac{1}{2} \ln \frac{\omega_{k, \alpha}}{\omega_{k, \beta}} \quad, \quad \alpha, \beta=1,2, A, B
\end{aligned}
$$

where $\omega_{k, \alpha} \equiv \sqrt{k^{2}+\mu_{\alpha}^{2}}$. We denote with $\mu_{A}$ and $\mu_{B}$ the arbitrary mass parameters while $\mu_{1} \equiv m_{1}$ and $\mu_{2} \equiv m_{2}$ are the physical masses. Note that $\rho_{12}^{\mathbf{k}}(t)=U_{\mathbf{k}}(t)$ and $\lambda_{12}^{\mathbf{k}}(t)=V_{\mathbf{k}}(t)$. We can now write the expansion of the flavor fields in the general form (we use a tilde to denote the generalized ladder operators):

$$
\phi_{\sigma}(x)=\int \frac{d^{3} k}{(2 \pi)^{\frac{3}{2}}}\left(u_{\mathbf{k}, \sigma}^{\phi}(t) \tilde{a}_{\mathbf{k}, \sigma}(t)+v_{-\mathbf{k}, \sigma}^{\phi}(t) \tilde{b}_{-\mathbf{k}, \sigma}^{\dagger}(t)\right) e^{i \mathbf{k} \cdot \mathbf{x}} \quad, \quad \sigma=A, B,
$$

which is to be compared with the expansion in the free field basis as given in Eqs. (6):

$$
\phi_{\sigma}(x)=\int \frac{d^{3} k}{(2 \pi)^{\frac{3}{2}}}\left(u_{\mathbf{k}, i}^{\phi}(t) a_{\mathbf{k}, \sigma}(t)+v_{-\mathbf{k}, i}^{\phi}(t) b_{-\mathbf{k}, \sigma}^{\dagger}(t)\right) e^{i \mathbf{k} \cdot \mathbf{x}}
$$

where $(\sigma, i)=(A, 1),(B, 2)$. The relation between the two sets of flavor operators is given as

$$
\begin{aligned}
& \left(\begin{array}{c}
\tilde{a}_{\mathbf{k}, \sigma}(t) \\
\tilde{b}_{-\mathbf{k}, \sigma}^{\dagger}(t)
\end{array}\right)=J^{-1}(t)\left(\begin{array}{c}
a_{\mathbf{k}, \sigma}(t) \\
b_{-\mathbf{k}, \sigma}^{\dagger}(t)
\end{array}\right) J(t)=\left(\begin{array}{cc}
\rho_{\sigma i}^{\mathbf{k} *}(t) & \lambda_{\sigma i}^{\mathbf{k}}(t) \\
\lambda_{\sigma i}^{\mathbf{k} *}(t) & \rho_{\sigma i}^{\mathbf{k}}(t)
\end{array}\right)\left(\begin{array}{c}
a_{\mathbf{k}, \sigma}(t) \\
b_{-\mathbf{k}, \sigma}^{\dagger}(t)
\end{array}\right), \\
& J(t)=\exp \left\{\int d^{3} k \xi_{\sigma, i}^{\mathbf{k}}\left[a_{\mathbf{k}, \sigma}^{\dagger}(t) b_{-\mathbf{k}, \sigma}^{\dagger}(t)-b_{-\mathbf{k}, \sigma}(t) a_{\mathbf{k}, \sigma}(t)\right]\right\},
\end{aligned}
$$

with $\xi_{\sigma, i}^{\mathbf{k}} \equiv \frac{1}{2} \ln \frac{\omega_{k, \sigma}}{\omega_{k, i}}$. For $\mu_{A}=m_{1}$ and $\mu_{B}=m_{2}$ one has $J=1$. Note that the transformation Eq.(36) is in fact a Bogoliubov transformation which leaves invariant the form $a_{\mathbf{k}, \sigma}^{\dagger}(t) a_{\mathbf{k}, \sigma}(t)-b_{-\mathbf{k}, \sigma}^{\dagger}(t) b_{-\mathbf{k}, \sigma}(t)$.

\section{THE CURRENTS FOR MIXED BOSON FIELDS}

Before presenting the exact oscillation formula, let us investigate in this Section the structure of currents and charges for the mixed fields. This will enable us to identify the relevant physical observables to look at for flavor oscillations. Since we are here interested in vacuum oscillations, in the following we neglect interaction terms and only consider the free field Lagrangian for two charged scalar fields with a mixed mass term:

$$
\mathcal{L}(x)=\partial_{\mu} \Phi_{f}^{\dagger}(x) \partial^{\mu} \Phi_{f}(x)-\Phi_{f}^{\dagger}(x) M \Phi_{f}(x),
$$

with $\Phi_{f}^{T}=\left(\phi_{A}, \phi_{B}\right), M=\left(\begin{array}{cc}m_{A}^{2} & m_{A B}^{2} \\ m_{A B}^{2} & m_{B}^{2}\end{array}\right)$. By means of Eq.(1)

$$
\Phi_{f}(x)=\left(\begin{array}{cc}
\cos \theta & \sin \theta \\
-\sin \theta & \cos \theta
\end{array}\right) \Phi_{m}(x),
$$

$\mathcal{L}$ becomes diagonal in the basis $\Phi_{m}^{T}=\left(\phi_{1}, \phi_{2}\right)$ :

$$
\mathcal{L}(x)=\partial_{\mu} \Phi_{m}^{\dagger}(x) \partial^{\mu} \Phi_{m}(x)-\Phi_{m}^{\dagger}(x) M_{d} \Phi_{m}(x),
$$

where $M_{d}=\operatorname{diag}\left(m_{1}^{2}, m_{2}^{2}\right)$ and $m_{A}^{2}=m_{1}^{2} \cos ^{2} \theta+m_{2}^{2} \sin ^{2} \theta, m_{B}^{2}=m_{1}^{2} \sin ^{2} \theta+m_{2}^{2} \cos ^{2} \theta, m_{A B}^{2}=\left(m_{2}^{2}-m_{1}^{2}\right) \sin \theta \cos \theta$.

The Lagrangian $\mathcal{L}$ is invariant under the global $U(1)$ phase transformations $\Phi_{m}^{\prime}=e^{i \alpha} \Phi_{m}$ : as a result, we have the conservation of the Noether charge $Q=\int d^{3} x I^{0}(x)$, which is indeed the total charge of the system (we have $I^{\mu}(x)=i \Phi_{m}^{\dagger}(x) \overleftrightarrow{\partial^{\mu}} \Phi_{m}(x)$ with $\left.\overleftrightarrow{\partial^{\mu}} \equiv \overrightarrow{\partial^{\mu}}-\overleftarrow{\partial^{\mu}}\right)$.

Let us now consider the $S U(2)$ transformation

$$
\Phi_{m}^{\prime}(x)=e^{i \alpha_{j} \tau_{j}} \Phi_{m}(x) \quad, \quad j=1,2,3,
$$


with $\alpha_{j}$ real constants, $\tau_{j}=\sigma_{j} / 2$ and $\sigma_{j}$ being the Pauli matrices. For $m_{1} \neq m_{2}$, the Lagrangian is not generally invariant under (41) and we obtain, by use of the equations of motion,

$$
\begin{gathered}
\delta \mathcal{L}(x)=-i \alpha_{j} \Phi_{m}^{\dagger}(x)\left[M_{d}, \tau_{j}\right] \Phi_{m}(x)=-\alpha_{j} \partial_{\mu} J_{m, j}^{\mu}(x), \\
J_{m, j}^{\mu}(x)=i \Phi_{m}^{\dagger}(x) \tau_{j} \overleftrightarrow{\partial^{\mu}} \Phi_{m}(x) \quad, \quad j=1,2,3 .
\end{gathered}
$$

The corresponding charges, $Q_{m, j}(t) \equiv \int d^{3} x J_{m, j}^{0}(x)$, close the $s u(2)$ algebra (at each time $t$ ). The Casimir operator $C_{m}$ is proportional to the total charge: $C_{m} \equiv\left[\sum_{j=1}^{3} Q_{m, j}^{2}(t)\right]^{\frac{1}{2}}=\frac{1}{2} Q$. Observe also that the transformation induced by $Q_{m, 2}(t)$,

$$
\Phi_{f}(x)=e^{-2 i \theta Q_{m, 2}(t)} \Phi_{m}(x) e^{2 i \theta Q_{m, 2}(t)}
$$

is just the mixing transformation Eq.(39). Thus $2 Q_{m, 2}(t)$ is the generator of the mixing transformations. Moreover, $Q_{m, \pm}(t) \equiv \frac{1}{2}\left(Q_{m, 1}(t) \pm i Q_{m, 2}(t)\right), Q_{m, 3}$, and $C_{m}$ are nothing but $S_{ \pm}(t), S_{3}$, and $S_{0}$, respectively, as introduced in Eqs.(9)-(11). From Eq.(42) we also see that $Q_{m, 3}$ and $C_{m}$ are conserved, consistently with Eqs.(14)-(15). Observe that the combinations

$$
\begin{aligned}
Q_{1,2} & \equiv \frac{1}{2} Q \pm Q_{m, 3} \\
Q_{i} & =\int d^{3} k\left(a_{\mathbf{k}, i}^{\dagger} a_{\mathbf{k}, i}-b_{-\mathbf{k}, i}^{\dagger} b_{-\mathbf{k}, i}\right) \quad, \quad i=1,2,
\end{aligned}
$$

are simply the conserved] (Noether) charges for the free fields $\phi_{1}$ and $\phi_{2}$ with $Q_{1}+Q_{2}=Q$.

We now perform the $S U(2)$ transformations on the flavor doublet $\Phi_{f}$ :

$$
\Phi_{f}^{\prime}(x)=e^{i \alpha_{j} \tau_{j}} \Phi_{f}(x) \quad, \quad j=1,2,3,
$$

and obtain:

$$
\begin{gathered}
\delta \mathcal{L}(x)=-i \alpha_{j} \Phi_{f}^{\dagger}(x)\left[M, \tau_{j}\right] \Phi_{f}(x)=-\alpha_{j} \partial_{\mu} J_{f, j}^{\mu}(x), \\
J_{f, j}^{\mu}(x)=i \Phi_{f}^{\dagger}(x) \tau_{j} \overleftrightarrow{\partial^{\mu}} \Phi_{f}(x) \quad, \quad j=1,2,3 .
\end{gathered}
$$

The related charges, $Q_{f, j}(t) \equiv \int d^{3} x J_{f, j}^{0}(x)$, still fulfil the $s u(2)$ algebra and $C_{f}=C_{m}=\frac{1}{2} Q$. Due to the offdiagonal (mixing) terms in the mass matrix $M, Q_{f, 3}(t)$ is time-dependent. This implies an exchange of charge between $\phi_{A}$ and $\phi_{B}$, resulting in the flavor oscillations. This suggests to us to define indeed the flavor charges as

$$
\begin{aligned}
Q_{A}(t) & \equiv \frac{1}{2} Q+Q_{f, 3}(t), \\
Q_{B}(t) & \equiv \frac{1}{2} Q-Q_{f, 3}(t),
\end{aligned}
$$

with $Q_{A}(t)+Q_{B}(t)=Q$. These charges have a simple expression in terms of the flavor ladder operators:

$$
Q_{\sigma}(t)=\int d^{3} k\left(a_{\mathbf{k}, \sigma}^{\dagger}(t) a_{\mathbf{k}, \sigma}(t)-b_{-\mathbf{k}, \sigma}^{\dagger}(t) b_{-\mathbf{k}, \sigma}(t)\right) \quad, \quad \sigma=A, B .
$$

This is because they are connected to the Noether charges $Q_{i}$ of Eq.(46) via the mixing generator: $Q_{\sigma}(t)=$ $G_{\theta}^{-1}(t) Q_{i} G_{\theta}(t)$, with $(\sigma, i)=(A, 1),(B, 2)$. Note that the flavor charges are invariant under the transformation Eq.(36).

\footnotetext{
${ }^{1}$ Note that, in absence of mixing, these charges would indeed be the flavor charges, being the flavor conserved for each generation.
} 


\section{THE OSCILLATION FORMULA FOR MIXED BOSONS}

Let us now calculate the oscillation formula for mixed bosons. We will first follow the approach of Ref. 12 and show that the oscillation formulas there presented exhibit a dependence on the arbitrary mass parameters $\mu_{\sigma}$, a feature which is not physically acceptable. We will do this by using the generalized operators introduced above. Then we will show how to cure this pathology, in analogy to what was done in Refs. [8, 9], where the exact formula for neutrino oscillations was derived and it was shown to be independent from the arbitrary mass parameters that can be introduced in the expansions of the flavor fields.

\section{A. The oscillation formula of Binger and $\mathbf{J i}$}

Following Ref. [12], let us define the (generalized) flavor state by acting on the mass vacuum $|0\rangle_{1,2}$ with the flavor creation operators (we omit momentum indices):

$$
\left|\widetilde{a}_{A}\right\rangle \equiv \widetilde{a}_{A}^{\dagger}|0\rangle_{1,2}=\rho_{A 1} \cos \theta\left|a_{1}\right\rangle+\rho_{A 2} \sin \theta\left|a_{2}\right\rangle .
$$

with $\left|a_{i}\right\rangle=a_{i}^{\dagger}|0\rangle$. As already discussed in Ref. [12, the flavor state so defined is not normalized and the normalization factor has to be introduced as

$$
\widetilde{\mathcal{N}}_{A} \equiv\left\langle\widetilde{a}_{A} \mid \widetilde{a}_{A}\right\rangle=\rho_{A 1}^{2} \cos ^{2} \theta+\rho_{A 2}^{2} \sin ^{2} \theta
$$

We have

$$
\left\langle\widetilde{a}_{A}\left|\widetilde{N}_{A}\right| \widetilde{a}_{A}\right\rangle=\left(\rho_{A 1}^{2}+\lambda_{A 1}^{2}\right) \cos ^{2} \theta+\left(\rho_{A 2}^{2}+\lambda_{A 2}^{2}\right) \sin ^{2} \theta .
$$

The oscillation formula then follow as:

$$
\left\langle\widetilde{a}_{A}(t)\left|\widetilde{N}_{A}\right| \widetilde{a}_{A}(t)\right\rangle=\left\langle\widetilde{a}_{A}\left|\widetilde{N}_{A}\right| \widetilde{a}_{A}\right\rangle-4 \frac{\rho_{A 1}^{2} \rho_{A 2}^{2}}{\widetilde{\mathcal{N}}_{A}} \sin ^{2} \theta \cos ^{2} \theta \sin ^{2}\left(\frac{\Delta E}{2} t\right)
$$

and a similar one for the expectation value of $\tilde{N}_{B}$. From the above, as announced, it is evident that these formulas explicitly depend on the (arbitrary) parameters $\mu_{\sigma}$ (see Eq.(31)). We also note that, for $\mu_{A}=m_{1}$ and $\mu_{B}=m_{2}$, one has $\rho_{A 1}=1, \lambda_{A 1}=0, \rho_{A 2}=U$ and $\lambda_{A 2}=V$. Consequently, Eqs.(55), (56) reduce respectively to Eqs.(18) and (20) of Ref. [12].

\section{B. The exact oscillation formula}

We now show how a consistent treatment of the flavor oscillation for bosons in QFT can be given which does not exhibit the above pathological dependence on arbitrary parameters.

There are two key points to be remarked. A general feature of field mixing is that the number operator for mixed particles is not a well-defined operator. It is so because the mixing transformations mix creation and annihilation operators and then the annihilation (creation) operators for flavor particles and antiparticles do not commute at different times (see Eqs.(21)-(24)). Moreover, the number operator does depend on the arbitrary mass parameters. Much care is therefore required in the use of the number operator. A second remark is that the flavor states are not to be defined by using the vacuum $|0\rangle_{1,2}$ : the flavor states so defined are in fact not normalized and the normalization factor Eq.(54) depends on the arbitrary mass parameters.

These two difficulties can be bypassed by using the remedy already adopted in Refs. [8. 9] for the case of fermions: the flavor states have shown to be consistently defined by acting with the flavor creation operators on the flavor vacuum. The observable quantities are then the expectation values of the flavor charges on the flavor states: the oscillation formulas thus obtained do not depend on the arbitrary mass parameters.

In the line of Refs. [8,9], let us now define? the state of the $a_{A}$ particle as $\left|\tilde{a}_{\mathbf{k}, A}\right\rangle_{A, B} \equiv \tilde{a}_{\mathbf{k}, A}^{\dagger}(0)|0\rangle_{A, B}$ and consider the expectation values of the flavor charges (52) on it (analogous results follow if one considers $\left|\tilde{a}_{\mathbf{k}, B}\right\rangle_{A, B}$ ). We obtain:

\footnotetext{
${ }^{2}$ In the following, we will work in the Heisenberg picture: this is particularly convenient in the present context since special care has to be taken with the time dependence of flavor states (see the discussion in Ref. (8)).
} 


$$
\widetilde{\mathcal{Q}}_{\mathbf{k}, \sigma}(t) \equiv_{A, B}\left\langle\widetilde{a}_{\mathbf{k}, A}\left|\widetilde{Q}_{\sigma}(t)\right| \widetilde{a}_{\mathbf{k}, A}\right\rangle_{A, B}=\left|\left[\widetilde{a}_{\mathbf{k}, \sigma}(t), \widetilde{a}_{\mathbf{k}, A}^{\dagger}(0)\right]\right|^{2}-\left|\left[\widetilde{b}_{-\mathbf{k}, \sigma}^{\dagger}(t), \widetilde{a}_{\mathbf{k}, A}^{\dagger}(0)\right]\right|^{2} \quad, \quad \sigma=A, B
$$

We also have ${ }_{A, B}\left\langle 0\left|\widetilde{Q}_{\mathbf{k}, \sigma}(t)\right| 0\right\rangle_{A, B}=0$ and $\widetilde{\mathcal{Q}}_{\mathbf{k}, A}(t)+\widetilde{\mathcal{Q}}_{\mathbf{k}, A}(t)=1$.

A straightforward direct calculation shows that the above quantities do not depend on $\mu_{A}$ and $\mu_{B}$, i.e.:

$$
{ }_{A, B}\left\langle\widetilde{a}_{\mathbf{k}, A}\left|\widetilde{Q}_{\mathbf{k}, \sigma}(t)\right| \widetilde{a}_{\mathbf{k}, A}\right\rangle_{A, B}={ }_{A, B}\left\langle a_{\mathbf{k}, A}\left|Q_{\mathbf{k}, \sigma}(t)\right| a_{\mathbf{k}, A}\right\rangle_{A, B} \quad, \quad \sigma=A, B
$$

and similar one for the expectation values on $\left|\tilde{a}_{\mathbf{k}, B}\right\rangle_{A, B}$. Eq. (58) is a central result of our work: it confirms that the only physically relevant quantities are the above expectation values of flavor charges. Note that expectation values of the number operator, of the kind ${ }_{A, B}\left\langle\widetilde{a}_{\mathbf{k}, A}\left|\widetilde{N}_{\sigma}(t)\right| \widetilde{a}_{\mathbf{k}, A}\right\rangle_{A, B}=\left|\left[\widetilde{a}_{\mathbf{k}, \sigma}(t), \widetilde{a}_{\mathbf{k}, A}^{\dagger}(0)\right]\right|^{2}$ and similar ones, do indeed depend on the arbitrary mass parameters, although the flavor states are properly defined (i.e. on the flavor Hilbert space). The cancellation of these parameters happens only when considering the combination of squared modula of commutators of the form Eq.(57) [ A similar cancellation occurs for fermions [9] with the sum of the squared modula of anticommutators.

Finally, the explicit calculation gives

$$
\begin{aligned}
\mathcal{Q}_{\mathbf{k}, A}(t) & =\left|\left[a_{\mathbf{k}, A}(t), a_{\mathbf{k}, A}^{\dagger}(0)\right]\right|^{2}-\left|\left[b_{-\mathbf{k}, A}^{\dagger}(t), a_{\mathbf{k}, A}^{\dagger}(0)\right]\right|^{2} \\
& =1-\sin ^{2}(2 \theta)\left[\left|U_{\mathbf{k}}\right|^{2} \sin ^{2}\left(\frac{\omega_{k, 2}-\omega_{k, 1}}{2} t\right)-\left|V_{\mathbf{k}}\right|^{2} \sin ^{2}\left(\frac{\omega_{k, 2}+\omega_{k, 1}}{2} t\right)\right], \\
\mathcal{Q}_{\mathbf{k}, B}(t) & =\left|\left[a_{\mathbf{k}, B}(t), a_{\mathbf{k}, A}^{\dagger}(0)\right]\right|^{2}-\left|\left[b_{-\mathbf{k}, B}^{\dagger}(t), a_{\mathbf{k}, A}^{\dagger}(0)\right]\right|^{2} \\
& =\sin ^{2}(2 \theta)\left[\left|U_{\mathbf{k}}\right|^{2} \sin ^{2}\left(\frac{\omega_{k, 2}-\omega_{k, 1}}{2} t\right)-\left|V_{\mathbf{k}}\right|^{2} \sin ^{2}\left(\frac{\omega_{k, 2}+\omega_{k, 1}}{2} t\right)\right] .
\end{aligned}
$$

Notice the negative sign in front of the $\left|V_{\mathbf{k}}\right|^{2}$ terms in these formulas, in contrast with the fermion case [8,9]: the boson flavor charge can assume also negative values. This fact points to the statistical nature of the phenomenon: it means that when dealing with mixed fields, one intrinsically deals with a many-particle system, i.e. a genuine field theory phenomenon. This situation has a strong analogy with Thermal Field Theory (i.e. QFT at finite temperature) [16], where quasi-particle states are ill defined and only statistical averages make sense. Of course, there is no violation of charge conservation for the overall system of two mixed fields.

The above formulas are obviously different from the usual quantum mechanical oscillation formulas, which however are recovered in the relativistic limit (i.e. for $|\mathbf{k}|^{2} \gg \frac{m_{1}^{2}+m_{2}^{2}}{2}$ ). Apart from the extra oscillating term (the one proportional to $\left|V_{\mathbf{k}}\right|^{2}$ ) and the momentum dependent amplitudes, the QFT formulas carry the remarkable information about the statistics of the oscillating particles: for bosons and fermions the amplitudes (Bogoliubov coefficients) are drastically different according to the two different statistics $\left(\left|U_{\mathbf{k}}\right|\right.$ and $\left|V_{\mathbf{k}}\right|$ are circular functions in the fermion case and hyperbolic functions in the boson case). This fact also fits with the above mentioned statistical nature of the oscillation phenomenon in QFT. Note also that our treatment is essentially non-perturbative and in this differs from other QFT approaches to particle mixing and oscillations (see for example Ref. [17] for a review).

In order to better appreciate the features of the QFT formulas, it is useful to plot the oscillating charge in time for sample values of the masses and for different values of the momentum (we use same units for masses and momentum). It is evident how the effect of the extra oscillating term is maximal at lower momenta (see Figs.2 and 3) and disappears for large $k$ (see Fig.4) where the standard oscillation pattern is recovered. In the following plots we use $T_{k}=\frac{4 \pi}{\omega_{k, 2}-\omega_{k, 1}}$ and assume maximal mixing.

\footnotetext{
${ }^{3}$ One may think it could make sense to take the expectation value of the flavor charges on states defined on the mass Hilbert space, as the ones defined in Eq.(53). A direct calculation however shows that this is not the case and these expectation values depends on the mass parameters: the conclusion is that one must use the flavor Hilbert space.
} 


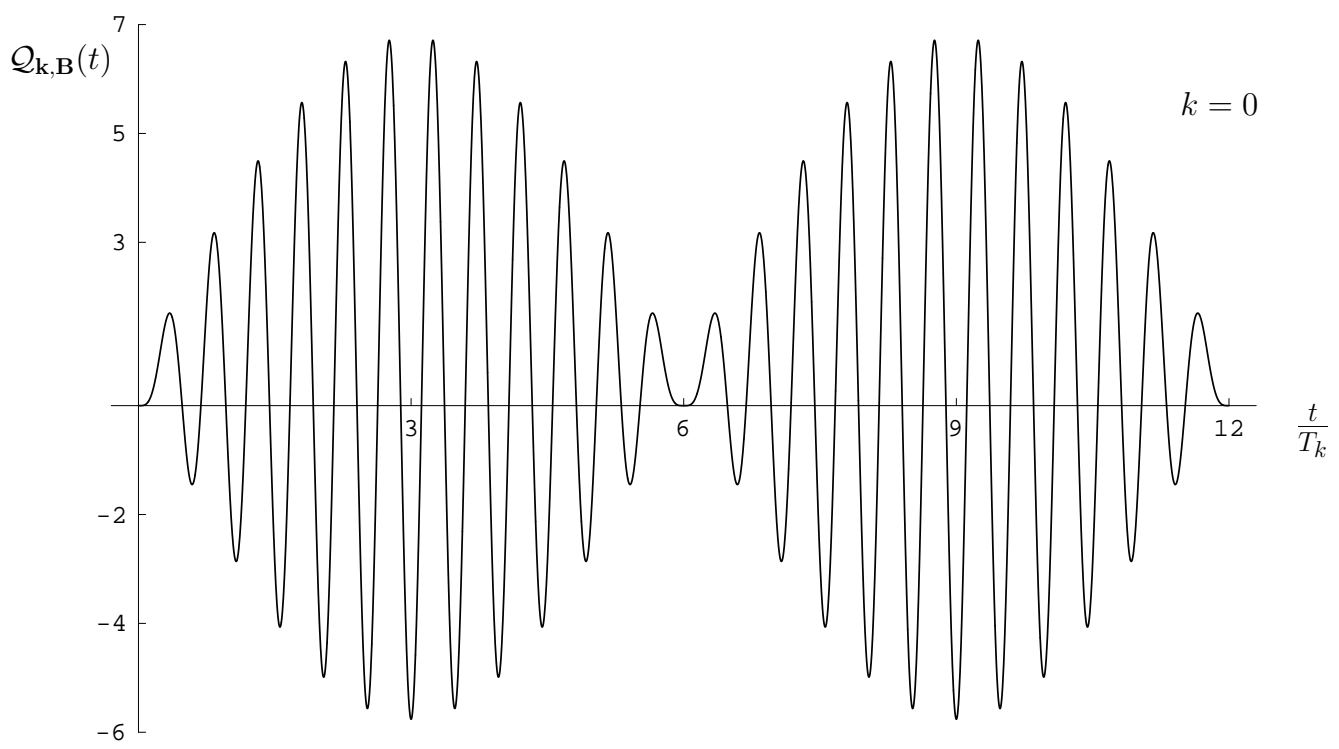

Figure 2: Plot of $\mathcal{Q}_{\mathbf{k}, B}(t)$ in function of time for $k=0, m_{1}=2, m_{2}=50$ and $\theta=\pi / 4$.

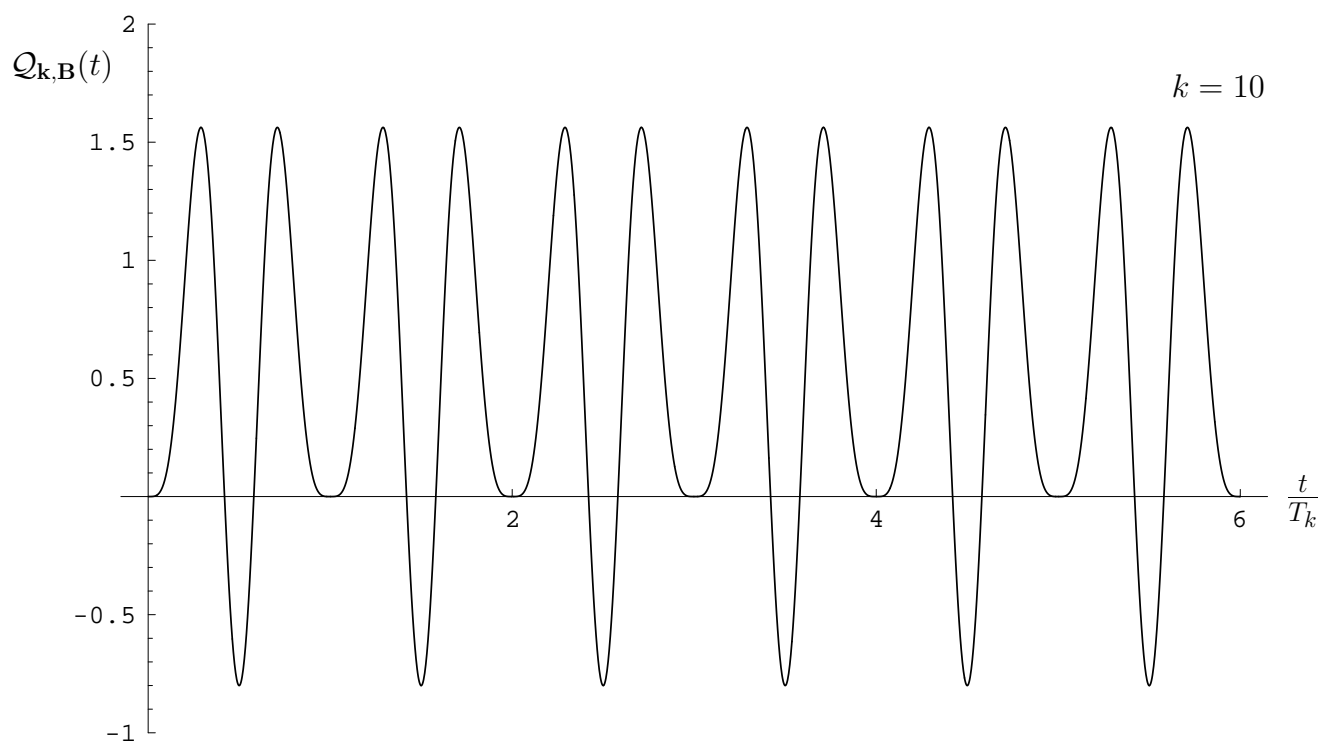

Figure 3: Plot of $\mathcal{Q}_{\mathbf{k}, B}(t)$ in function of time for $k=10, m_{1}=2, m_{2}=50$ and $\theta=\pi / 4$. 


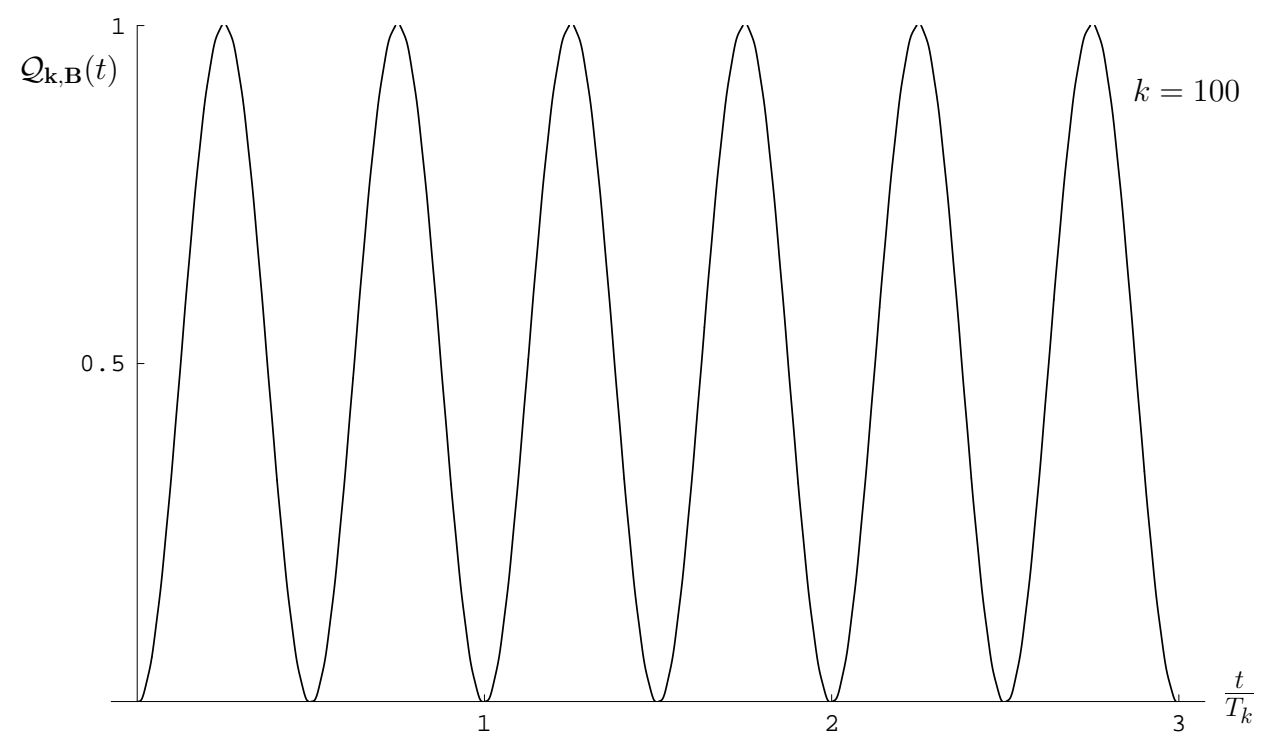

Figure 4: Plot of $\mathcal{Q}_{\mathbf{k}, B}(t)$ in function of time for $k=100, m_{1}=2, m_{2}=50$ and $\theta=\pi / 4$.

It is also interesting to plot the time average of the oscillating charge, $\overline{\mathcal{Q}}_{\mathbf{k}, B}=\frac{1}{n T_{k}} \int_{0}^{n T_{k}} d t \mathcal{Q}_{\mathbf{k}, B}(t)$, as a function of the momentum. In Figure 5 we plot $\mathcal{Q}_{\mathbf{k}, B}(t)$ averaged over two different time intervals, i.e. for $n=10$ and $n=100$ : it is interesting to observe how the larger is the time interval, the more the curve converges to the average of the standard formula, which has the value $\frac{1}{2}$. The behavior for large $\mathrm{k}$ is due to the fact that, as already observed, the exact oscillation formula reduces to the quantum mechanical oscillation one in the large momentum limit (i.e. for $\left.|k|^{2} \gg \frac{m_{1}^{2}+m_{2}^{2}}{2}\right)$.

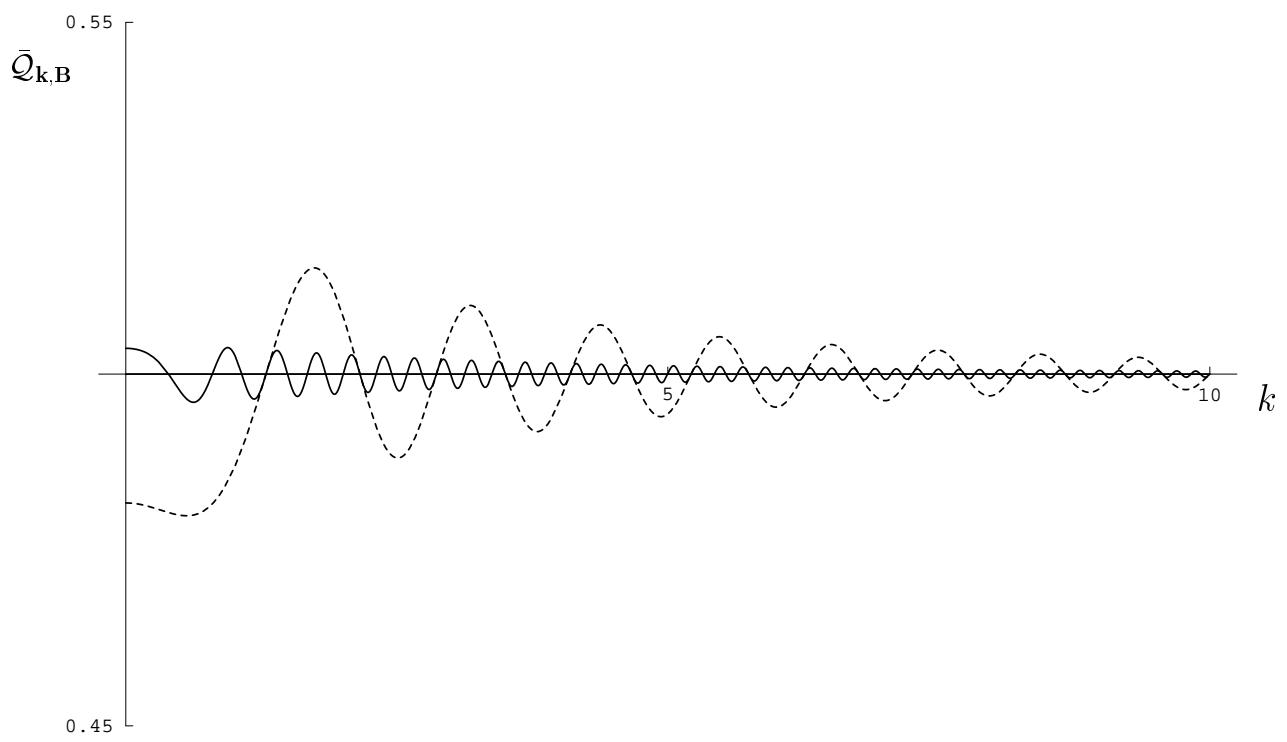

Figure 5: The time average of $\mathcal{Q}_{\mathbf{k}, B}(t)$ over $10 T_{k}$ (dashed line) and over $100 T_{k}$ (solid line) with respect to the average of the standard oscillation formula (horizontal axis) as a function of $k$ for the values $m_{1}=2, m_{2}=50$ and $\theta=\pi / 4$. 


\section{CONCLUSIONS}

In this paper we have considered the quantum field theoretical formulation of spin-zero boson field mixing and obtained the exact oscillation formula which does not depend on arbitrary mass parameters which can be introduced in full generality in the theory. We have also studied the structure of the currents and charges for the mixed fields. In order to make our discussion more transparent, we neglected the instability of the oscillating particles. This does not affect the general validity of our result which rests on the intrinsic features of QFT.

A crucial point in our analysis is the disclosure of the fact that the space for the mixed field states is unitarily inequivalent to the state space where the unmixed field operators are defined. This is a common feature with the QFT structure of the fermion mixing, which has recently been established [5 9]). The vacuum for the mixed fields turns out to be a generalized $S U(2)$ coherent state. Of course, in the boson case the condensate structure for the "flavor" vacuum is found to be very much different from the one in the fermion case. Besides the intrinsic mathematical interest, our analysis provides interesting phenomenological insights. It leads to the exact oscillation formula for bosons which predicts oscillation behaviors susceptible of being experimentally tested.

In fact, in the framework of the QFT analysis of Refs. [5, 11], a study of the meson mixing and oscillations has been already carried out in Ref. [12] and applied to the $\eta-\eta^{\prime}$ oscillation. However, the results of Ref. 12 give observable quantities which are dependent on arbitrary mass parameters, and this is of course physically not acceptable, as observed in Ref. [7]. In the present paper we have pointed out the origin of such a pathology and have shown how to obtain results which are independent from arbitrary parameters. The oscillation formula obtained in Ref. [12] has to be actually replaced with the exact one here presented. In order to compare our results with the ones of Ref. [12], we presented in Fig. 6 a plot of charge oscillations for $\eta-\eta^{\prime}$ at zero momentum, in correspondence to what has been done in Ref. 12].

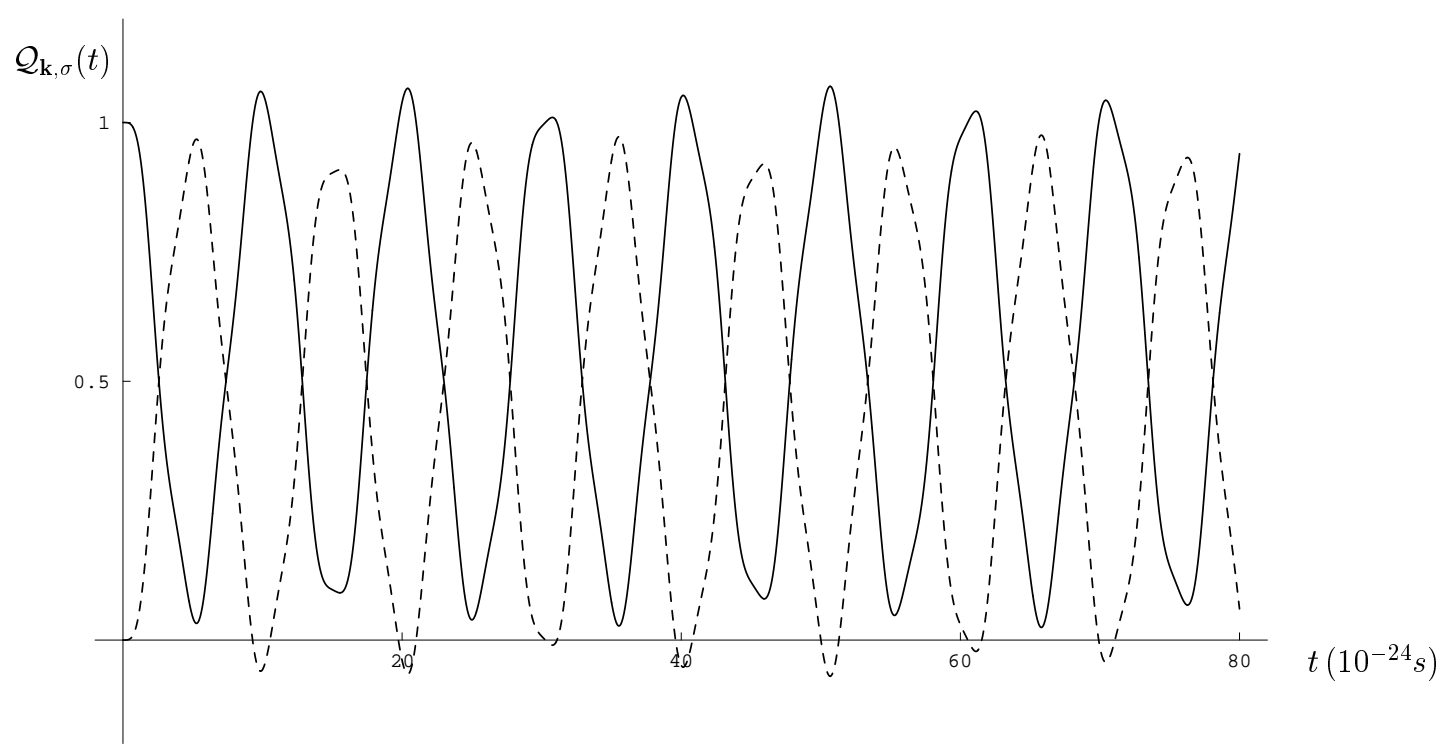

Figure 6: Plot of $\mathcal{Q}_{\mathbf{k}, \eta}(t)$ (solid line) and $\mathcal{Q}_{\mathbf{k}, \eta^{\prime}}(t)$ (dashed line) in function of time for an initially pure $\eta$ state and for $k=0, m_{1}=549 \mathrm{MeV}, m_{2}=958 \mathrm{MeV}$ and $\theta=-54^{\circ}$.

Let us close by observing that although our QFT analysis discloses features which cannot be ignored in any further study of the field mixing and oscillations, since they are intrinsic to the structure of the QFT formalism, nevertheless there are many aspects which are not fully understood and many features of the physics of mixing are still obscure [1], as already observed in the introduction. The mixing of neutrinos and their oscillations seem to be now experimentally established and quark mixing and meson mixing are widely accepted and verified. However, several questions 18 25] are the object of active discussion in the framework of the quantum mechanics formalism for neutrino oscillations. As a matter of fact, such a state of affairs has been a strong motivation for our searching in the structural aspects of QFT a possible hint to the understanding of particle mixing and oscillations. 


\section{ACKNOWLEDGMENTS}

We thank MURST, INFN and ESF for partial support.

\section{Appendix: Orthogonality between mass and flavor vacua}

We calculate here ${ }_{1,2}\langle 0 \mid 0(\theta, t)\rangle_{A, B}$. In the following we work at finite volume (discrete $k$ ) and suppress the time dependence of the operators when $t=0$. Let us first observe that $|0(\theta, t)\rangle_{A, B}=e^{i H t}|0(\theta, 0)\rangle_{A, B}$, with $H=\sum_{i=1}^{2} \sum_{\mathbf{k}} \omega_{k, i}\left(a_{\mathbf{k}, i}^{\dagger} a_{\mathbf{k}, i}+b_{-\mathbf{k}, i}^{\dagger} b_{-\mathbf{k}, i}\right)$. Thus we have ${ }_{1,2}\langle 0 \mid 0(\theta, t)\rangle_{A, B}={ }_{1,2}\langle 0 \mid 0(\theta, 0)\rangle_{A, B}$.

We then define $\prod_{\mathbf{k}} f_{0}^{\mathbf{k}}(\theta) \equiv \prod_{\mathbf{k}}{ }_{1,2}\left\langle 0\left|G_{\mathbf{k}, \theta}^{-1}(0)\right| 0\right\rangle_{1,2}$ and observe that

$$
\begin{aligned}
\frac{d}{d \theta} f_{0}^{\mathbf{k}}(\theta) & =\left|V_{\mathbf{k}}\right|_{1,2}\left\langle 0\left|\left(b_{-\mathbf{k}, 1} a_{\mathbf{k}, 2}+b_{-\mathbf{k}, 2} a_{\mathbf{k}, 1}\right) G_{\mathbf{k}, \theta}^{-1}\right| 0\right\rangle_{1,2} \\
& =-\left|V_{\mathbf{k}}\right|_{1,2}\left\langle 0\left|G_{\mathbf{k}, \theta}^{-1}\left(a_{\mathbf{k}, 2}^{\dagger} b_{-\mathbf{k}, 1}^{\dagger}+a_{\mathbf{k}, 1}^{\dagger} b_{-\mathbf{k}, 2}^{\dagger}\right)\right| 0\right\rangle_{1,2}
\end{aligned}
$$

where, we recall, $\left|V_{\mathbf{k}}\right| \equiv V_{\mathbf{k}}(0)$ in our notation of Section II. We now consider the identity

$$
\begin{aligned}
\left(b_{-\mathbf{k}, 1} a_{\mathbf{k}, 2}+b_{-\mathbf{k}, 2} a_{\mathbf{k}, 1}\right) G_{\mathbf{k}, \theta}^{-1} & =G_{\mathbf{k}, \theta}^{-1} G_{\mathbf{k},-\theta}^{-1}\left(b_{-\mathbf{k}, 1} a_{\mathbf{k}, 2}+b_{-\mathbf{k}, 2} a_{\mathbf{k}, 1}\right) G_{\mathbf{k},-\theta} \\
& =G_{\mathbf{k}, \theta}^{-1}\left[b_{-\mathbf{k}, A}(-\theta) a_{\mathbf{k}, B}(-\theta)+b_{-\mathbf{k}, B}(-\theta) a_{\mathbf{k}, A}(-\theta)\right] .
\end{aligned}
$$

Then the equation follows

$$
\begin{aligned}
\frac{d}{d \theta} f_{0}^{\mathbf{k}}(\theta) & =-2\left|V_{\mathbf{k}}\right|^{2} \cos \theta \sin \theta f_{0}^{\mathbf{k}}(\theta)+\sin ^{2} \theta\left|V_{\mathbf{k}}\right|^{3}{ }_{1,2}\left\langle 0\left|G_{\mathbf{k}, \theta}^{-1}\left(a_{\mathbf{k}, 2}^{\dagger} b_{-\mathbf{k}, 1}^{\dagger}+a_{\mathbf{k}, 1}^{\dagger} b_{-\mathbf{k}, 2}^{\dagger}\right)\right| 0\right\rangle_{1,2} \\
& =-2\left|V_{\mathbf{k}}\right|^{2} \cos \theta \sin \theta f_{0}^{\mathbf{k}}(\theta)-\sin ^{2} \theta\left|V_{\mathbf{k}}\right|^{2} \frac{d}{d \theta} f_{0}^{\mathbf{k}}(\theta)
\end{aligned}
$$

and

$$
\frac{d}{d \theta} f_{0}^{\mathbf{k}}(\theta)=-\frac{2\left|V_{\mathbf{k}}\right|^{2} \cos \theta \sin \theta}{1+\sin ^{2} \theta\left|V_{\mathbf{k}}\right|^{2}} f_{0}^{\mathbf{k}}(\theta),
$$

which is solved by

$$
f_{0}^{\mathbf{k}}(\theta)=\frac{1}{1+\sin ^{2} \theta\left|V_{\mathbf{k}}\right|^{2}},
$$

with the initial condition $f_{0}^{\mathbf{k}}(0)=1$.

We observe that we can operate in a similar fashion directly with $f_{0}^{\mathbf{k}}(\theta, t) \equiv{ }_{1,2}\left\langle 0\left|G_{\mathbf{k}, \theta}^{-1}(t)\right| 0\right\rangle_{1,2}$. We then find that $f_{0}^{\mathbf{k}}(\theta, t)$ is again given by Eq. [66) and thus it is actually time-independent. We also note that by a similar procedure it can be proved that $\lim _{V \rightarrow \infty}{ }_{A, B}\left\langle 0(\theta, t) \mid 0\left(\theta^{\prime}, t\right)\right\rangle_{A, B} \rightarrow 0$ for $\theta^{\prime} \neq \theta$.

[1] M. Zralek, Acta Phys. Polon. B29 (1998) 3925 hep-ph/9810543.

[2] Super-Kamiokande Collaboration (Y. Fukuda et al.) Phys. Rev. Lett. 81 (1998) 1562; Kamiokande Collaboration(S. Hatakeyama et al.), Phys. Rev. Lett. 81 (1998) 2016.

[3] T. Cheng and L. Li, Gauge Theory of Elementary Particle Physics, Clarendon Press, Oxford, 1989;

N. Cabibbo, Phys. Rev. Lett. 10 (1963) 531; M. Kobayashi and T. Maskawa, Prog. Theor. Phys. 49 (1973) 652. 
[4] H. Fritzsch and Z. Xing, Prog. Part. Nucl. Phys. 45 (2000) 1 hep-ph/9912358. H. Fritzsch and Z. Xing, Phys. Rev. D 57 (1998) 594; Phys. Lett. B 413 (1997) 396

R. Barbieri, P. Creminelli and A. Romanino, Nucl. Phys. B559 (1999) 17 hep-ph/9903460. R. Barbieri, L. J. Hall, G. L. Kane and G. G. Ross, hep-ph/9901228].

[5] M. Blasone and G. Vitiello, Annals Phys. 244 (1995) 283 hep-ph/9501263].

[6] K. C. Hannabuss and D. C. Latimer, J. Phys. AA33 (2000) 1369.

[7] K. Fujii, C. Habe and T. Yabuki, Phys. Rev. D59 (1999) 113003 hep-ph/9807266; K. Fujii, C. Habe and T. Yabuki, hep-ph/0102001.

[8] M. Blasone, P. A. Henning and G. Vitiello, Phys. Lett. B451 (1999) 140 [hep-th/9803157]; M. Blasone, in "Erice 1998, From the Planck length to the Hubble radius" 584-593, hep-ph/9810329;

[9] M. Blasone and G. Vitiello, Phys. Rev. D60 (1999) 111302 hep-ph/9907382].

[10] S. M. Bilenky and B. Pontecorvo, Phys. Rep. 41 (1978) 225 ; S. M. Bilenkii and S. T. Petcov, Rev. Mod. Phys. 59 (1987) 671.

[11] M. Blasone, P.A. Henning and G. Vitiello, in "La Thuile 1996, Results and perspectives in particle physics" $139-152$ hep-ph/9605335.

[12] M. Binger and C. Ji, Phys. Rev. D60 (1999) 056005 hep-ph/9901407.

[13] D. Lurie, Particles and Fields, (Interscience Publ., 1968).

[14] A. Perelomov, Generalized Coherent States and Their Applications, (Springer-Verlag, Berlin, 1986).

[15] C. Itzykson and J. B. Zuber, Quantum Field Theory, (McGraw-Hill, New York, 1980);

N. N. Bogoliubov. A. A. Logunov, A. I. Osak and I. T. Todorov, General Principles of Quantum Field Theory, (Kluwer Academic Publishers, Dordrech, 1990).

[16] H. Umezawa, H. Matsumoto and M. Tachiki, Thermo Field Dynamics and Condensed States, (North-Holland Publ.Co., Amsterdam, 1982) ;

H. Umezawa, Advanced Field Theory: Micro, Macro and Thermal Physics (American Institute of Physics, 1993).

[17] M. Beuthe, hep-ph/0010054].

[18] Y. Grossman and H. J. Lipkin, Phys. Rev. D 55 (1997) 2760 hep-ph/9607201.

[19] H. J. Lipkin, Phys. Lett. B348 (1995) 604 hep-ph/9501269.

[20] Y. Srivastava, A. Widom and E. Sassaroli, Eur. Phys. J. C2 (1998) 769 ; Y. Srivastava, A. Widom and E. Sassaroli, Z. Phys. C66 (1995) 601; Y. N. Srivastava, A. Widom and E. Sassaroli, Phys. Lett. B344 (1995) 436.

[21] J. Lowe, B. Bassalleck, H. Burkhardt, A. Rusek, G. J. Stephenson and T. Goldman, Phys. Lett. B384 (1996) 288 [hep$\mathrm{ph} / 9605234$.

[22] K. Kiers, S. Nussinov and N. Weiss, Phys. Rev. D 53 (1996) 537 hep-ph/9506271.

[23] H. Burkhardt, J. Lowe, G. J. Stephenson and T. Goldman, Phys. Rev. D 59 (1999) 054018 hep-ph/9803365.

[24] C. Giunti and C. W. Kim, hep-ph/0011074].

[25] Y. Takeuchi, Y. Tazaki, S. Y. Tsai and T. Yamazaki, Mod. Phys. Lett. A14 (1999) 2329. 